\title{
Comparison of time domain numerical solvers for the propagation of a Gaussian pulse inside a rectangular waveguide
}

$$
\text { V. Vegh* I. W. Turner }{ }^{\dagger}
$$

(Received March 2002; revised 5 June 2002)

\begin{abstract}
We analyse techniques for increasing the accuracy and efficiency of a finite-volume time-domain (FV-TD) cell-centred computational methodology. Various state-of-the-art spatial and temporal discretisation methods employed to solve Maxwell equations on multi-dimensional structured grid networks are investigated and the dispersive and dissipative errors inherent in those techniques examined. Both staggered and unstaggered grid approaches are considered. Staggered and unstaggered Leapfrog and Runge-Kutta time integration methods are analysed by the use of Gaussian microwave
\end{abstract}

*School of Mathematical Sciences, Queensland University of Technology, Brisbane, Australia. mailto:v.vegh@qut.edu.au

†School of Mathematical Sciences, Queensland University of Technology, Brisbane, Australia mailto:i.turner@qut.edu.au

${ }^{0}$ See http://anziamj.austms.org.au/V44/CTAC2001/Vegh for this article, (C) Austral. Mathematical Soc. 2003. Published 1 April 2003. ISSN 1446-8735 
pulse simulations. The implementation of typical electromagnetic boundary conditions is also deliberated. Finally, a comparison of the classical finite-difference time-domain (FD-TD) method and FV-TD numerical results for a standard case study in rectangular waveguides allows the accuracy of the developed methods to be assessed.

\section{Contents}

1 Introduction

C782

2 Cell-centred finite-volume time-domain methods

C786

3 Boundary conditions

C792

4 Results

C794

5 Conclusion

C799

References

C800

\section{Introduction}

Many computational methodologies have been developed to solve Maxwell's Equations for a variety of important applications in Science and Engineering [1, 2, 3, 4]. Although microwaves are frequently used for heating purposes, in this paper only microwave pulses are analysed for the purpose of radar and signal processing. Over the last two decades, research work in Computational Electromagnetics (CEM) has seen the development of approximations both for the integral and point forms of the governing Maxwell's 
equations. In this work, the traditional staggered in time and space FD-TD [5] scheme is compared to a number of cell-centred finitevolume time-domain approaches (ccFV-TD). Most of the schemes discussed here are formulated for generalised unstructured applications. However, this part of the research work is applied on a simpler structured mesh case study, to allow the numerical results to be compared directly to the FD-TD solution.

Staggered schemes store approximations of the components of the electromagnetic fields at different spatial locations within the computational domain. For example, FD-TD uses the Yee lattice shown in Figure 1 to achieve that objective. In this case, the electric and magnetic fields are staggered also in time in order to stabilise the explicit time marching scheme. On the contrary, cell-centred schemes store all of the electromagnetic fields at the same spatial location (the cell centre). Typically, cell-centred schemes are much easier to manage and implement (in terms of algorithm planning, design and storage data structures) in comparison with their staggered counterparts. Note however that even though the electric and magnetic field components are evaluated at the same locations inside a given cell, it is still possible to stagger in time schemes which are cell-centred in space. These are usually classified as staggered and unstaggered leapfrog integrations in time, where the electric and magnetic field components are either stored at different locations or at the same location in time, respectively. In this work, third and fourth order Runge-Kutta (RK3 and RK4) methods are implemented also on the cell-centred in space approach to analyse their performance against the Leapfrog time marching schemes.

The problem studied here concerns a $T E_{10}$ Gaussian pulse propagated longitudinally in a typical rectangular waveguide. The end of the waveguide is short-circuited, and the reflected waves are absorbed in the scattered field region of the waveguide. Initially, a 


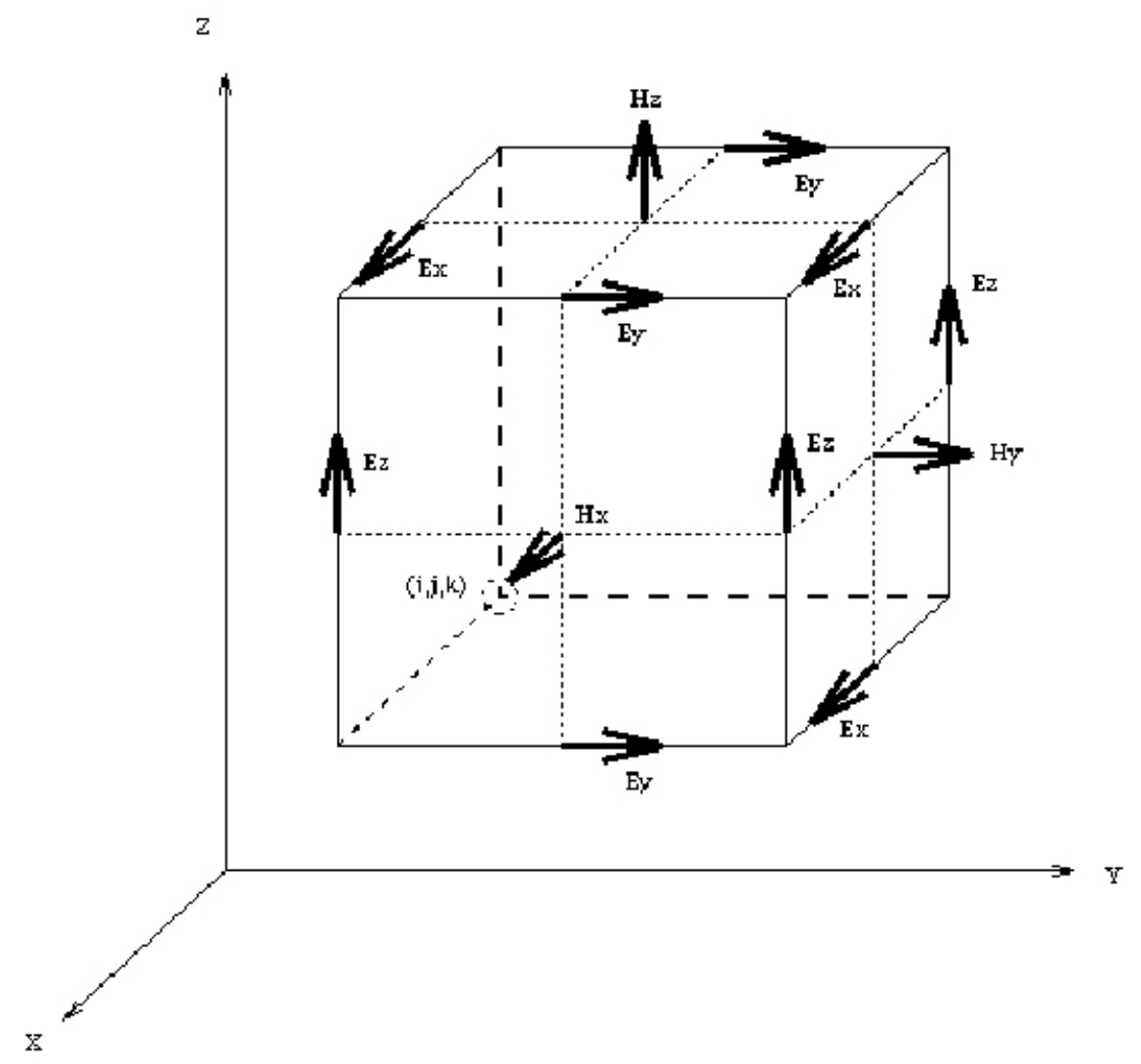

Figure 1: A Yee cell. The components of the electric (E) and magnetic $(\mathbf{H})$ fields are stored at different locations along the cell edges and on the cell faces, respectively. For the FD-TD algorithm, the electric and magnetic fields are also staggered half a time step apart. 
detailed description of the mathematical formulation is provided for the time domain cell-centred in space methods, and typical boundary condition implementation for the conducting walls, input plane and absorbing boundary layer is deliberated. In the scattered field region of the waveguide, an existing Perfectly Matched Layer (PML) staggered in space methodology [6] is adapted to the cell-centred approaches to absorb any reflected impinging waves in the scattered field region of the waveguide.

Primarily, the study of a propagating Gaussian pulse in an empty waveguide allows the performance of the different Maxwell's equations time domain solvers to be analysed under free space conditions. The pulse presents a good test for the FV-TD methods, and highlights the evident dissimilarities between the different cell-centred schemes, and shows also the differences when compared to the FDTD method. Depending on the spatial and temporal discretisation methods utilised, the schemes exhibit both dispersive and dissipative numerical errors. Dissipative errors cause the loss of wave amplitude, whereas dispersive errors affect the wave propagation speed. These errors accumulate and need to be monitored for a number of time steps.

This paper consists of five sections. In the next section the mathematical formulation for discretising Maxwell's equations using a finite-volume approach is presented, highlighting the different cell-centred schemes. Then there follows a detailed discussion of the electromagnetic boundary condition implementation. In the proceeding section the results for the empty waveguide case study are presented, and comparisons between the different methods are provided via a number of graphical illustrations that elucidate the dissipative and dispersive nature of the different schemes. Finally, the conclusions of this work are summarised. 


\section{Cell-centred finite-volume time-domain methods}

For numerical simulation using a finite-volume methodology, the point form of the Maxwell's equations (1) must be recast into a discrete volumetric form:

$$
\begin{gathered}
\nabla \times \mathbf{E}=-\frac{\partial \mathbf{B}}{\partial t}, \quad \nabla \times \mathbf{H}=\frac{\partial \mathbf{D}}{\partial t}+\mathbf{J}, \\
\mathbf{B}=\mu_{0} \mathbf{H}, \quad \mathbf{D}=\epsilon \mathbf{E}, \quad \mathbf{J}=\sigma \mathbf{E}, \quad \epsilon=\epsilon_{0} \epsilon^{\prime}, \quad \sigma=\omega \epsilon_{0} \epsilon^{\prime \prime},
\end{gathered}
$$

where, $\mathbf{E}$ is the electric field, $\mathbf{H}$ is the magnetic field, $\mathbf{D}$ is the electric flux, $\mathbf{B}$ is the magnetic flux, $\mathbf{J}$ is the current density, $\mu_{0}$ is the permeability of free-space, $\epsilon_{0}$ is the permittivity of free-space, $\epsilon$ is the permittivity, $\epsilon^{\prime}$ is the relative electric constant, $\epsilon^{\prime \prime}$ is the relative loss factor, $\sigma$ is the electric conductivity and $\omega=2 \pi f$ where $f$ is the input frequency. Note that for most microwave heating problems, the media are dielectric and current free. To arrive at the volumetric form, we integrate over a discrete finite volume cell. Unlike previous techniques where the integral was approximated using Stokes' theorem [3, 7], hence the Divergence theorem is applied to the volumetric representation to obtain a surface volume relationship between the electric and magnetic fields. The continuum equation is then cast into discrete form

$$
\begin{aligned}
\frac{\partial \mathbf{B}_{p}}{\partial t} & =-\frac{1}{\Delta V} \sum_{F \in \zeta_{p}} \mathbf{n} \times \mathbf{E}_{F} \Delta S_{F}, \\
\frac{\partial \mathbf{D}_{p}}{\partial t} & =\frac{1}{\Delta V} \sum_{F \in \zeta_{p}} \mathbf{n} \times \mathbf{H}_{F} \Delta S_{F}-\mathbf{J}_{p},
\end{aligned}
$$

where $\mathbf{n}$ is the unit outward normal through a face of a particular cell (see Figure 2). In equation (2), $\zeta_{p}$ is the set of faces that constitute the $p$ th cell in a computational domain, and $\Delta S_{F}$ and $\Delta V$ 


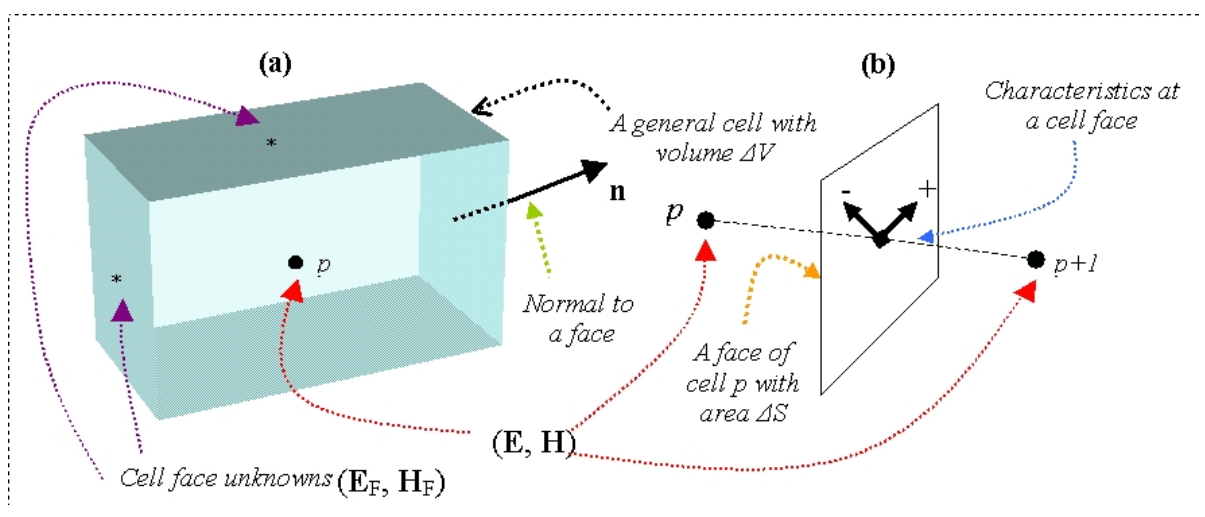

Figure 2: A general cell within the computational domain showing the locations of the unknowns.

are the surface area of a particular face in $\zeta_{p}$ and the volume of the $p$ th cell, respectively. For the above discrete representation of Maxwell's equations (2), the surface integral approximation is second order in space if $\mathbf{E}_{F}$ and $\mathbf{H}_{F}$ are the values at the midpoint of the face. By visiting all of the cells that constitute the solution domain, a system of ordinary differential equations (ODEs) results. A number of methods are proposed to resolve the system of ODEs.

When approximating the differential operator in time, the discretisation can introduce either dispersion or dissipation errors [4]. Numerous techniques can be utilised to resolve (2) into time discrete form. In [8] a number of approaches similar to the FD-TD solution methodology were investigated. For a function $\phi$, equations (3) and (4) are the staggered (SLF) and unstaggered (ULF) leapfrog discretisations respectively, which are both $\mathcal{O}\left(\Delta t^{2}\right)$ approximations:

$$
{\frac{\partial \phi^{n+\frac{1}{2}}}{\partial t}}^{=} \frac{\phi^{n+1}-\phi^{n}}{\Delta t},
$$




$$
\frac{\partial \phi^{n}}{\partial t}=\frac{\phi^{n+1}-\phi^{n-1}}{2 \Delta t} .
$$

Methods using the discretisations in (3) have been used to numerically solve for the electromagnetic fields governed by Maxwell's equations. Using (3), the equations of (2) are written in discrete form

$$
\begin{gathered}
\mathbf{H}_{p}^{n+\frac{1}{2}}=\mathbf{H}_{p}^{n-\frac{1}{2}}-\frac{\Delta t}{\mu_{0} \Delta V} \sum_{F \in \zeta_{p}} \mathbf{n} \times \mathbf{E}_{F}^{n} \Delta S_{F}, \\
\mathbf{E}_{p}^{n+1}=\frac{2 \epsilon-\sigma \Delta t}{2 \epsilon+\sigma \Delta t} \mathbf{E}_{p}^{n}-\frac{2 \Delta t}{(2 \epsilon+\sigma \Delta t) \Delta V} \sum_{F \in \zeta_{p}} \mathbf{n} \times \mathbf{H}_{F}^{n+\frac{1}{2}} \Delta S_{F},
\end{gathered}
$$

Similarly, (2) with the substitution of the discretisation in (4) becomes

$$
\begin{gathered}
\mathbf{H}_{p}^{n+1}=\mathbf{H}_{p}^{n-1}-\frac{2 \Delta t}{\mu_{0} \Delta V} \sum_{F \in \zeta_{p}} \mathbf{n} \times \mathbf{E}_{F}^{n} \Delta S_{F}, \\
\mathbf{E}_{p}^{n+1}=\frac{\epsilon-\sigma \Delta t}{\epsilon+\sigma \Delta t} \mathbf{E}_{p}^{n-1}-\frac{2 \Delta t}{(\epsilon+\sigma \Delta t) \Delta V} \sum_{F \in \zeta_{p}} \mathbf{n} \times \mathbf{H}_{F}^{n} \Delta S_{F},
\end{gathered}
$$

Equations (5) to (8) require the approximation to the electric and magnetic fields at the cell faces. It is possible to propose a number of interpolating and extrapolating schemes to approximate these cell face unknowns. On structured grids, the simplest way to approximate the unknowns at a cell face is by averaging the cell values about a particular face. Such a technique imposes second order in space and time for (5-8). These particular finite volume methods, Leapfrog discretisations, will be referred to as: SLF (5-6), staggered in time and unstaggered in space; and ULF (7-8), unstaggered in space and unstaggered in time. 
The averaging about a cell face demonstrates the simplest way to approximate the terms inside the summations in (5) to (8). At the $p$ th cell in equation (2), Intensity-Vector Splitting (IVS) [4], which is a concept that originated from Computational Fluid Dynamics (CFD), is applied to replace the $\mathbf{n} \times \mathbf{E}_{F}$ and $\mathbf{n} \times \mathbf{H}_{F}$ terms. The notion behind IVS is to include an extra term in the flux facial expression to dampen any numerical oscillations, and to capture any discontinuities (such as shocks and contact surfaces in CFD) in the solution. At a cell face $F$, equations (9) and (10) depict the derived form of the IVs result:

$$
\begin{aligned}
& \mathbf{n} \times \mathbf{E}_{F}=\frac{1}{2} \mathbf{n} \times\left(\mathbf{E}_{F}^{+}+\mathbf{E}_{F}^{-}\right)+\frac{1}{2} \mathbf{n} \times\left([\mu c]^{+} \mathbf{n} \times \mathbf{H}_{F}^{+}-[\mu c]^{-} \mathbf{n} \times \mathbf{H}_{F}^{-}\right), \\
& \mathbf{n} \times \mathbf{H}_{F}=\frac{1}{2} \mathbf{n} \times\left(\mathbf{H}_{F}^{+}+\mathbf{H}_{F}^{-}\right)-\frac{1}{2} \mathbf{n} \times\left([\epsilon c]^{+} \mathbf{n} \times \mathbf{E}_{F}^{+}-[\epsilon c]^{-} \mathbf{n} \times \mathbf{E}_{F}^{-}\right),
\end{aligned}
$$

where, the wave speed is given by $c=1 / \sqrt{\mu \epsilon}$. It is evident from equations (9) and (10) that the \pm characteristics have to be approximated for a given cell face (see Figure 2). Note: the IVS result cannot be implemented with (3) since the values for the magnetic and electric fields are required at different time levels. For this reason, time marching schemes that allow the electric and magnetic field components to be located at the same instant in time have to be employed. Numerical experimentation has shown that IVS when incorporated with (4) incurs large errors due to the time discretisation of the electric and magnetic fields [4]. Therefore, higher order time stepping methods are to be implemented. The following third order Runge-Kutta (RK3) method was implemented with the IVs scheme discussed above:

$$
\phi^{n+\frac{1}{3}}=\phi^{n}+\frac{\Delta t}{3} \frac{\partial \phi^{n}}{\partial t}, \quad \phi^{n+\frac{2}{3}}=\phi^{n}+\frac{2 \Delta t}{3}{\frac{\partial \phi^{n+\frac{1}{3}}}{\partial t}},
$$




$$
\phi^{n+1}=\phi^{n}+\frac{\Delta t}{4}\left(3{\frac{\partial \phi^{n+\frac{2}{3}}}{\partial t}}^{2}+\frac{\partial \phi^{n}}{\partial t}\right) .
$$

The outlined RK3 scheme is the one commonly used in CEM, and hence it was chosen to solve the equations of (2) that employ (9) and (10). Also, a fourth order Runge-Kutta (RK4) method was implemented:

$$
\begin{aligned}
& \hat{\phi}^{n+\frac{1}{2}}=\phi^{n}+\frac{\Delta t}{2} \frac{\partial \phi^{n}}{\partial t}, \\
& \tilde{\phi}^{n+\frac{1}{2}}=\phi^{n}+\frac{\Delta t}{2}{\frac{\partial \hat{\phi}^{n+\frac{1}{2}}}{\partial t}}, \\
& \bar{\phi}^{n+1}=\phi^{n}+\Delta t{\frac{\partial \tilde{\phi}^{n+\frac{1}{2}}}{\partial t}}^{,}
\end{aligned}
$$

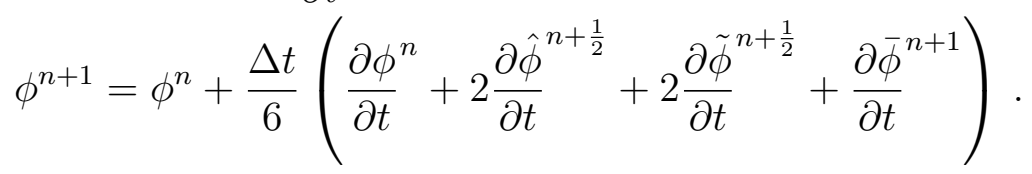

Substituting the IVS result into (2) and by applying the RK solvers of (11) and (12), the RK3-IVS and RK4-IVS techniques are obtained, respectively. Note that equation (2) can be solved using the RK3 and RK4 solvers without the inclusion of damping, and this suggestion is pursued later in this section. As shown in Figure 2, the \pm characteristics for the IVS are required at a particular cell face. Different strategies can be proposed and derived to approximate the values at the cell face joining any two adjacent cells. The simplest of these is the zeroth order substitution for an unknown $\xi$ :

$$
\xi_{p+\frac{1}{2}}^{-}=\xi_{p}, \quad \xi_{p+\frac{1}{2}}^{+}=\xi_{p+1} .
$$

Imposing the zeroth order approximation in RK3-IVS and RK4-IVS leads to a first order in space and third order in time RK3-1-IVs 
numerical method, and a first order in space and fourth order in time RK4-1-IVS numerical method respectively. On a structured grid, a general linear extrapolation model that is second order in space can also be derived:

$$
\xi_{p+\frac{1}{2}}^{-}=\frac{1}{2}\left(3 \xi_{p}-\xi_{p-1}\right), \quad \xi_{p+\frac{1}{2}}^{+}=\frac{1}{2}\left(3 \xi_{p+1}-\xi_{p+2}\right) .
$$

Using the approximations of (14), the RK3-2L-IVS and RK4-2LIVS methods are developed, which are simultaneously the third and fourth order in time and second order in space linear extrapolation numerical schemes. By using discrete data points within the vicinity of the cell faces, it is possible to find least squares gradient approximations at the cell centres [9]. Equation (15) is a truncated representation of the Taylor series:

$$
\delta \mathbf{r} \cdot \nabla \xi(\mathbf{r}) \approx \xi(\mathbf{r}+\delta \mathbf{r})-\xi(\mathbf{r}) .
$$

The above formula yields a system of linear equations that is cast into matrix form as $\mathbf{A} \cdot \nabla \xi_{p}=\mathbf{d}$. The gradient that minimises $\left\|\mathbf{A} \cdot \nabla \xi_{p}-\mathbf{d}\right\|^{2}$ with respect to the inner product on $\mathbb{R}^{k}$ is obtained by solving the normal equations. The value of $k$ equals the number of neighbouring nodes utilised to obtain the gradient at the $p$ th cell. In this paper, the gradients were constructed using only the adjacent cells of $\zeta_{p}$ (that is $k=6$ ). As a consequence, the gradients of the electric and magnetic fields are used to approximate the \pm characteristics at the cell faces. Using these gradients, equation (16) establishes a second order approximation for the \pm states:

$$
\xi_{p+\frac{1}{2}}^{-}=\xi_{p}+\delta^{\prime} \cdot \nabla \xi_{p}, \quad \xi_{p+\frac{1}{2}}^{+}=\xi_{p+1}+\delta^{\prime \prime} \cdot \nabla \xi_{p+1} .
$$

In equation (16), $\delta^{\prime}$ and $\delta^{\prime \prime}$ are the vectors from the nodes $p$ to $p+\frac{1}{2}$ and $p+1$ to $p+\frac{1}{2}$, respectively (see Figure 2). Subsequently, the methods that employ (16) are classified as RK3-2G-IVS and RK4-2GIVS, depending on the time integration. The various IVs methods 
can also be implemented without flux splitting and are referred to here as the RK3(RK4)-2L and RK3(RK4)-2G methods, which are the third (fourth) order linear extrapolation and the spatial gradient approximations, respectively.

\section{Boundary conditions}

In this section, the perfectly conducting wall, the incident field and absorbing boundary conditions are treated. On a perfectly conducting wall, the following conditions need to be satisfied [10]:

$$
\mathbf{n} \times \mathbf{E}=\mathbf{0}, \quad \mathbf{n} \cdot \mathbf{H}=0 .
$$

On structured grids, (17) implies that the tangential components of the electric field at a perfectly conducting wall boundary are zero. Equation (17) also entails that the magnetic components that are normal to that conducting wall are continuous across that wall. For the implementation of the incident field boundary condition, a classical waveguide is depicted in Figure 3. Typically, a fictitious dielectric is introduced inside the scattered region to absorb any reflected backward travelling waves. In Figure 3 it is assumed that $z_{0}$ represents the location where the incident field $I$ is located. The scatter field $S$ is the region between 0 and $z_{0}$, and the full field $F$ occupies the region between $z_{0}$ and $d$. The scattered and full field regions have length $l_{1}=z_{0}$ and $l_{2}=d-z_{0}$, respectively. At the input plane, the incident field is introduced using $F=S+I$ and a continuous $T E_{10}$ incident field is implemented as an input boundary condition. Across the input plane, the scattered and full fields are computed according to the following incident field assumptions:

$$
E_{y}^{I}=E_{0} \sin \frac{\pi x}{a} \cos \left(\omega t-\beta_{0} z_{0}\right),
$$




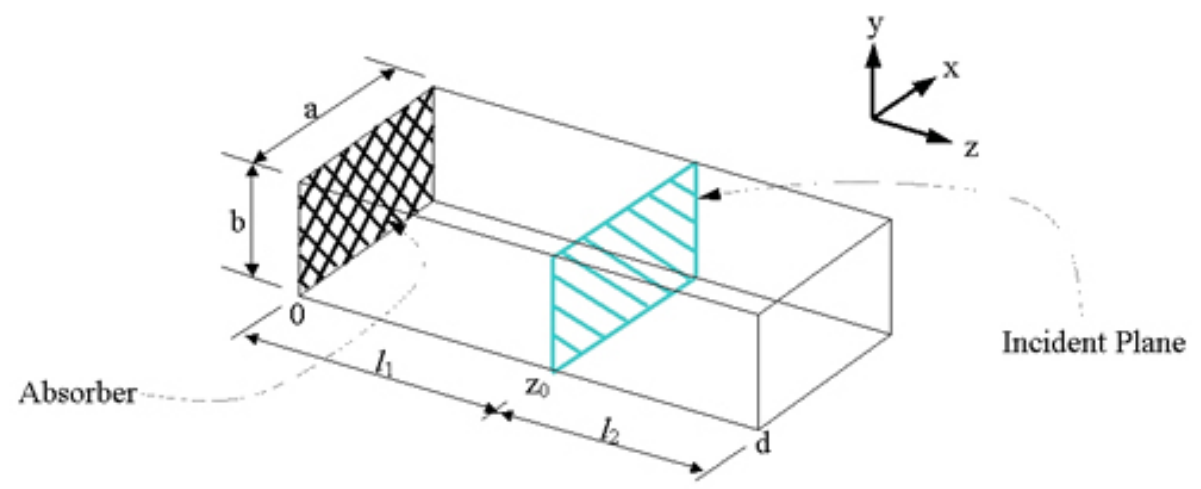

Figure 3: A waveguide with incident and absorbing boundary conditions.

$$
\begin{gathered}
H_{x}^{I}=-\frac{\beta_{0}}{\omega \mu_{0}} E_{0} \sin \frac{\pi x}{a} \cos \left(\omega t-\beta_{0} z_{0}\right), \\
H_{z}^{I}=-\frac{\pi}{\omega \mu_{0} a} E_{0} \cos \frac{\pi x}{a} \sin \left(\omega t-\beta_{0} z_{0}\right), \\
\beta_{0}=\sqrt{\omega^{2} \mu_{0} \epsilon_{0}-\left(\frac{\pi}{a}\right)^{2}} .
\end{gathered}
$$

In Figure 3, an absorbing boundary layer is represented inside the scatter field region of the waveguide. A previously proposed Perfectly Matched Layer (PML) boundary condition [6] has been used to absorb any waves inside the scattered filed region of the waveguide. In this work, this boundary condition has been adapted for the use in the cell-centred schemes discussed in the previous section. The PML boundary condition is based on matching the impedance of the absorbing medium to the impedance of free-space. The PML boundary conditions are based on the following augmented Maxwell's equations:

$$
\frac{\partial \mathbf{B}}{\partial t}+\mathbf{J}^{*}=\nabla \times \mathbf{E}, \quad \frac{\partial \mathbf{D}}{\partial t}+\mathbf{J}=-\nabla \times \mathbf{H},
$$


where

$$
\mathbf{J}^{*}=\sigma^{*} \mathbf{H}, \quad \sigma^{*}=\frac{\mu_{0}}{\epsilon_{0}} \sigma .
$$

By satisfying (22), the impedance of the PML equals that of freespace, and no reflections occur. From the reformulation of the Maxwell's equations, (1) is replaced by (21) in the discretisations, and new discrete in space equations are obtained for the numerical simulation of the electromagnetic behaviour within the absorbing material. Specific implementation details of the PML absorber will be outlined in future work, which will demonstrate the cell-centred finite-volume time-domain methods for the purpose of microwave heating.

\section{Results}

The waveguide of Figure 3 is used as a case study to numerically simulate a Gaussian microwave pulse inside the apparatus. The full field region of the waveguide is truncated via a short-circuit, and the scattered field region employs the Petropoulos type PML boundary condition to absorb any backward travelling waves. The dimension of the waveguide is $a=0.1 \mathrm{~m}, b=0.05 \mathrm{~m}$ and $d=$ $0.4 \mathrm{~m}$. At $z_{0}=0.1 \mathrm{~m}$, the incident plane is excited using a $T E_{10}$ $2.45 \mathrm{GHz}$ electromagnetic wave, with an average input power of $1 \mathrm{~W}$. The $T E_{10}$ wave is multiplied by a Gaussian function to propagate a microwave pulse inside the waveguide:

$$
\operatorname{Gauss}(t)=\exp \left[\left(-\frac{2.5 t}{T}-1\right)^{2}\right] .
$$

In equation (23), $T$ represents the wave period inside the waveguide. The instantaneous electromagnetic fields were monitored over two 
periods. The domain of the waveguide is discretised into 54,000 $(30 \times 15 \times 120)$ Cartesian cells. The time stepping of the numerical solvers is constrained by the relationship:

$$
\Delta t=\frac{0.9}{c_{\max } \sqrt{\frac{1}{\delta x^{2}}+\frac{1}{\delta y^{2}}+\frac{1}{\delta z^{2}}}},
$$

where, $c_{\max }$ is the maximum expected wave speed in the waveguide, and $\delta x, \delta y$ and $\delta z$ are the minimum mesh dimensions in the $x, y$ and $z$ coordinate directions [2]. Figures 4 and 5 show the results for the simulated $T E_{10}$ Gaussian pulse using the numerical techniques discussed throughout the previous sections. Since it is well known that the FD-TD scheme is highly accurate, all of the established cell-centred numerical techniques have been compared to the FDTD method to demonstrate the relative accuracy of each scheme. It is evident from the figures that the unstaggering of the unknowns introduces errors, and hence, higher order time marching schemes are required to better capture the FD-TD solution.

In the proceeding paragraphs, specific comments regarding the accuracy of the schemes presented in Section 2 and exhibited in Figures 4 and 5 are given. The introduction of intensity vector splitting smooths the noise that is apparent in the ULF, SLF and RK3(RK4)$2 \mathrm{G}$ techniques (see Figures $4 \mathrm{a}, 4 \mathrm{~b}, 4 \mathrm{c}$ and $5 \mathrm{c}$ ). It is well known that using higher order time integration techniques requires more computational effort to resolve the unknowns, and consequently, the techniques that employ IVS with RK3 and RK4 time integration are more computationally intensive than the FD-TD method. In this work, only the accuracy of the different cell centred schemes are compared, an analysis of the times to compute the numerical solutions has been left for future investigations. See in the figures that the methods that make use of the gradients to approximate the \pm characteristics at the cell faces can capture the pulse as well as any of the other cell-centred numerical schemes discussed (see 
Figures $4 \mathrm{c}-\mathrm{d}$ and $5 \mathrm{c}-\mathrm{d}$ ). Note that the results or RK3(RK4)-2L-IVS have not been shown, since they were comparable to those obtained using RK4-2G-IVS. In the numerical solutions it was observed also that results for RK3-2L were comparable to the RK4-2L scheme, and as a consequence, only the RK4-2L results are illustrated.

From the figures it is evident that the adaptation of IVS in the schemes (see Figures 4d, 5b and 5d) smooth the results; although note that the RK4-2L scheme has apparent smoothing also. This is because the approximation uses a second order linear extrapolation, which removes any rapid changes or oscillations in the numerical time marching. In Figure $4 a$ observe that the ULF scheme is conditionally unstable [4]. Figure 5b highlights that the RK4-1-IVS scheme, which utilises the zeroth order extrapolation for the \pm characteristics, is highly dissipative as observed by Liu [4]. Furthermore, the first order spatial discretisation inadequately captures the behaviour of the solution. However, note that such methods are able to capture the wave phase, but the amplitude is smeared considerably.

In Figures 4c-d and 5c-d observe that the numerical solution is out of phase with the FD-TD benchmark solution. In Figure 5a the RK4-2L scheme, which is suitable only for structured grids, appears to capture the wave phase. These findings are an artifact of the spatial discretisation, and to further investigate how the phase errors could be reduced, higher order spatial discretisations would have to be adapted to the previously outlined cell-centred schemes. The ULF, SLF, RK3(RK4)-2L and RK4-1-IVS techniques exhibit substantial wave front amplitude errors (see Figures $4 \mathrm{a}-\mathrm{b}$ and $5 \mathrm{a}-\mathrm{b}$ ). For the RK3(RK4)-2L method this is because of the grid used in the approximation of the cell face unknowns is four grid cells wide. Hence, as the incident field is propagated, the apparent noise due to the introduction of the microwave energy is propagated with the pulse. 

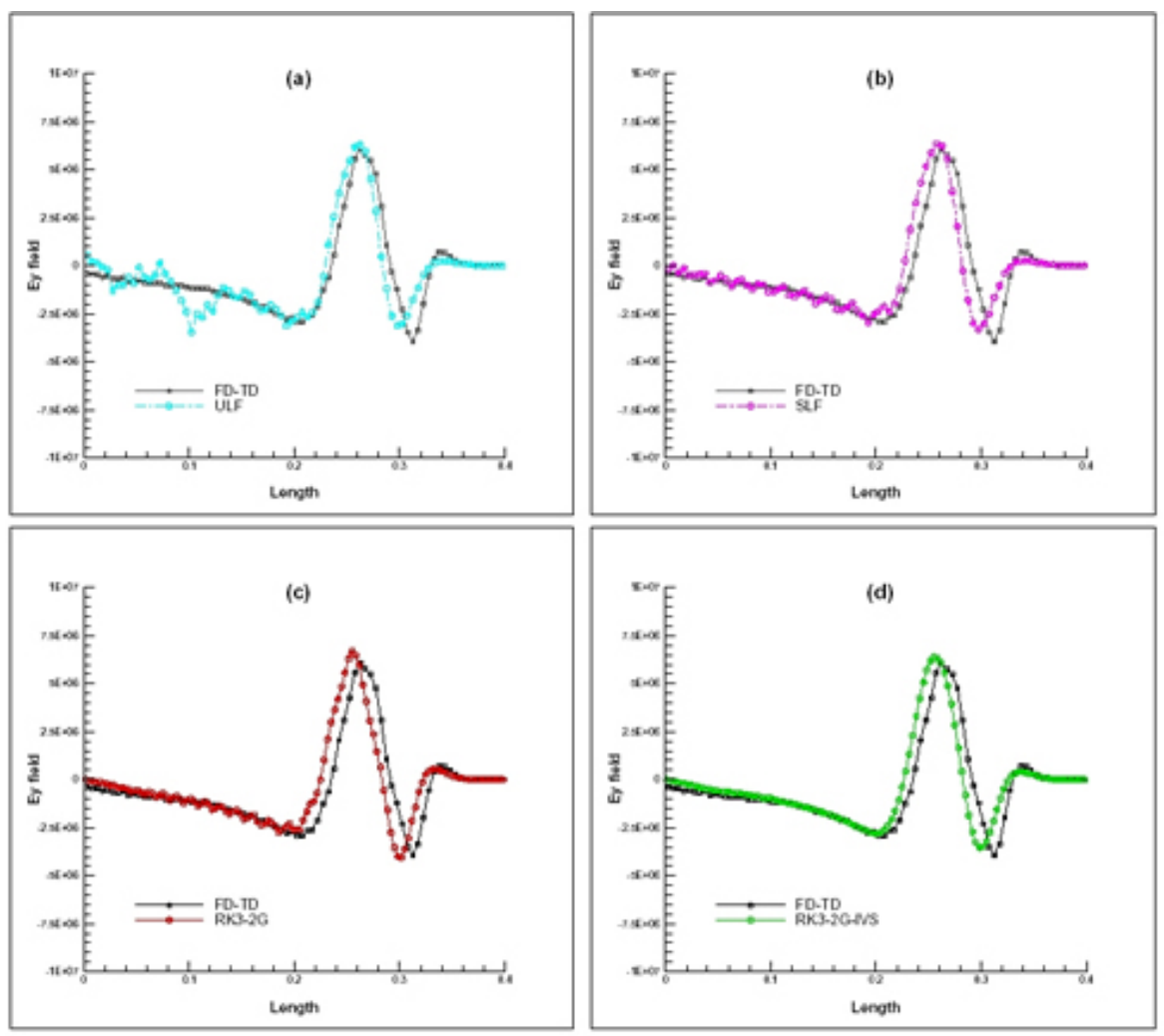

Figure 4: A two period pulse inside a $T E_{10}$ waveguide showing time versus the $y$-coordinate of the Electric field $(\mathrm{V} / \mathrm{m})$; (a) ULF; (b) SLF; (c) RK3-2G and (d) RK3-2G-IVS. 

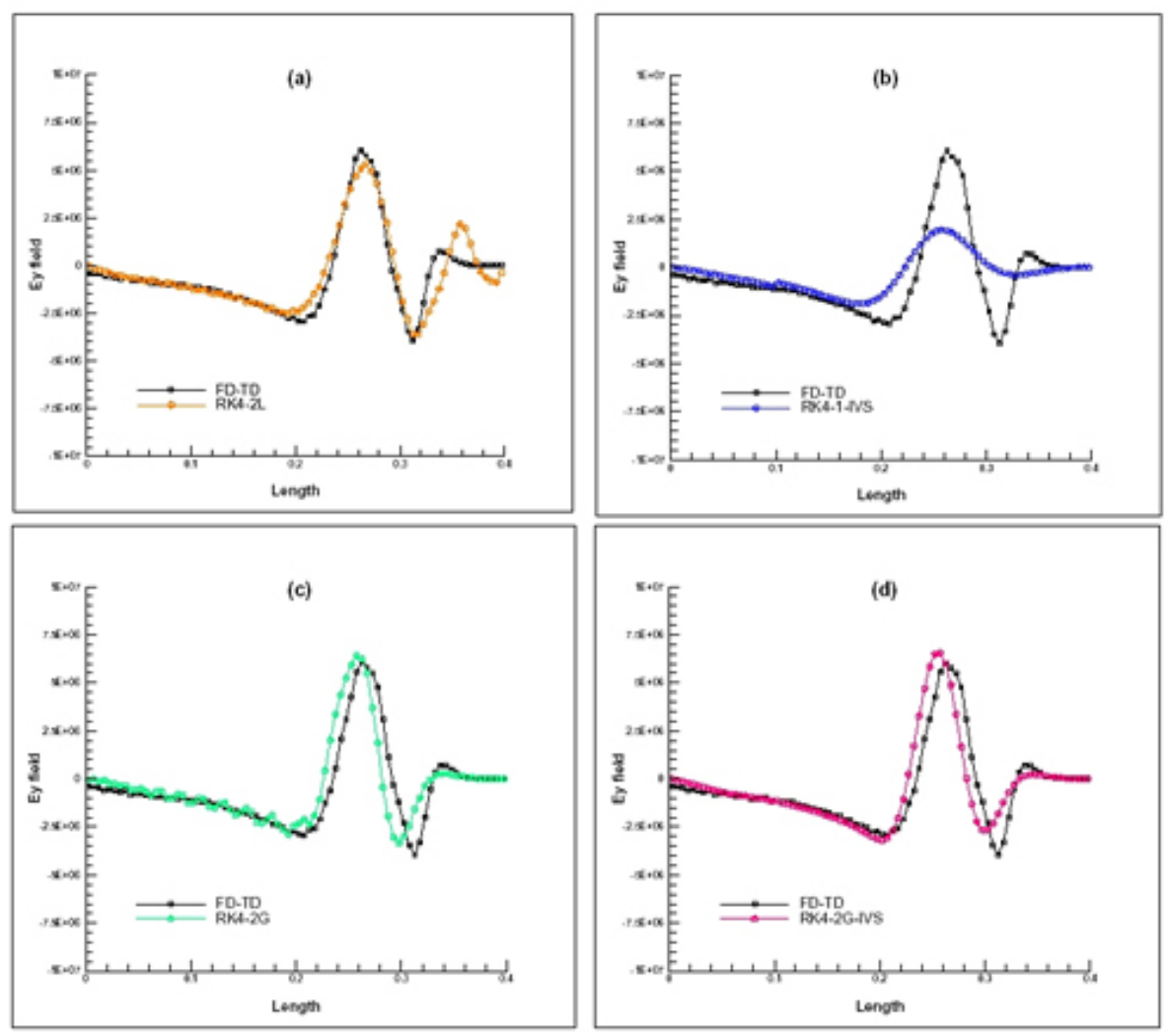

Figure 5: A two period pulse inside a $T E_{10}$ waveguide showing time versus the $y$-coordinate of the Electric field $(\mathrm{V} / \mathrm{m})$; (a) RK42L; (b) RK4-1-IVS; (c) RK4-2G and (d) RK4-2G-IVS. 


\section{Conclusion}

Cell centred time domain solvers for the Maxwell's equations were investigated and a number of solution strategies have been applied to resolve the Gaussian pulse waveguide study. The governing equations were discretised using non-traditional techniques to obtain a surface-volume representation that was solved numerically using a number of different numerical strategies. The FD-TD method is very accurate, but it is not easily migrated to unstructured domains. A number of cell-centred schemes have been formulated for unstructured domains, but were implemented here only on structured grids so that they could be compared to the classical FD-TD method. Further research in the area will demonstrate how these schemes can be implemented on completely unstructured grids.

Leapfrog staggered (SLF) and unstaggered (ULF) time integration techniques were used to time march the discrete surface-volume representation of Maxwell's equations. When intensity vector splitting (IVS) was introduced into the RK3(RK4)-2G schemes, the results appeared to be smooth, and the evident noise in the schemes was removed. This reduction in noise was due to the damping that these techniques induced in the numerical solution. The work clearly showed that time domain cell-centred Maxwell's equations numerical solvers have produced reasonable results for the SLF scheme. It was observed also that the Runge-Kutta based methods, with and without intensity vector splitting, produced results that were comparable to the classical FD-TD method.

For the purpose of microwave heating, future research will analyse in more detail the effect that a lossy material can have on the electromagnetic phenomenon evolving on the grid. In that work, more rigorous tests are to be conducted regarding dispersion and dissipation errors. 
Acknowledgements: The authors thank Dr. Huawei (Bob) Zhao for his input into the development of this paper. Also, many thanks to Dr. Joseph Young for his help with the visualisations in the accompanying presentation.

\section{References}

[1] D. C. Dibben and A. C. Metaxas. Time Domain Finite Element Analysis of Multimode Microwave Applicators Loaded with Low and High Loss Materials. Intl. Proceedings 5th Microwave and High Frequency Heating Conf., St John's College, Cambridge, pp. A3.1-A3.4, September 1995. C782

[2] H. Zhao and I. Turner. An analysis of the finite-difference time-domain method for modelling the microwave heating of dielectric materials within a three-dimensional cavity system. Journal of Microwave Power and Electromagnetic Energy, 1996. 31(4): p. 199-214. C782, C795

[3] N. K. Madsen and R. W. Ziolkowski. A three-dimensional modified finite volume technique for Maxwell's equations. Electromagnetics, 1990(10): pp. 147-161. C782, C786

[4] Y. Liu. Fourier Analysis of Numerical Algorithms for the Maxwell Equations. Journal of Computational Physics, 1996. 124: pp. 396-416. C782, C787, C789, C796

[5] K. S. Yee. Numerical Solution of Initial Boundary Value Problem Involving Maxwell's Equations in Isotropic Media. IEEE Trans. Antennas Propagat., 1966. 14: pp. 302-307. C783 
[6] P. G. Petropoulos, L. Zhao, and A. C. Cangellaris. A Reflectionles Sponge Layer Absorbing Boundary Condition for the Solution of Maxwell's Equations with High-Order Staggered Finite Difference Schemes. Journal of Computational Physics, 1998. 139: pp. 184-208. C785, C793

[7] N. K. Madsen and R. W. Ziolkowski. Numerical solution of Maxwells' equations in the time domain using irregular nonorthogonal grids. Wave Motion, 1988(10): pp. 583-596. C786

[8] A. Taflove. Re-inventing Electromagnetics: Supercomputing Solution of Maxwell's Equations Via Direct Time Integration on Space Grids. AIAA paper 92-0333, 1992(New York). C787

[9] T. J. Barth. Aspects of Unstructured Grids and Finite-Volume Solvers for the Euler and Navier-Stokes Equations. Lecture Notes Presented at the KI Lecture Series, 1994-05, 1995. C791

[10] R. E. Collin. Field Theory of Guided Waves. 1960: McGraw-Hill Book Company. C792 